\title{
OPERATIONAL EXPERIENCE OF THE ATLAS ACCELERATOR ${ }^{*}$
}

F. K. Den Hartog, J. M. Bogaty, L. M. Bollinger, B. E. Clifft, S. L. Craig, R. E. Harden, P. Markovich, F. H. Munson, J. M. Nixon, R. C. Pardo, D. R. Phillips, K. W. Shepard, I. R. Tilbrook, and G. P. Zinkann Physics Division. Argonne National Laboratory Argonne, IL 60439-4843 USA Abstract

The ATLAS accelerator consists of a HVEC model FN tandem accelerator injecting into a linac of independently-phased niobium superconducting resonators. The accelerator provides beams with masses from $6 \leq \mathrm{A} \leq 127$ and with energies ranging up to $20 \mathrm{MeV} / \mathrm{A}$ for the lightest ions and $4 \mathrm{MeV} / \mathrm{A}$ for the heaviest ions. Portions of the linac have been in operation since 1978 and, over the last decade, more than 35,000 hours of operating experience have been accumulated. The long-term stability of niobium resonators, and their feasibility for use in heavy-ion accelerators is now well established.

*Work supported by the U. S. Department of Energy, Nuclear Physics Division, under Contract W-31-109-Eng-38

\section{DISCLAIMER}

This report was prepared as an account of work sponsored by an agency of the United States Government. Neither the United States Government nor any agency thereof, not any of their employees, makes any warranty, express or implied, or assumes any legal liability or responsibility for the accuracy, completeness, or usefulness of any information, apparatus, product, or process disclosed, or represir:'s that its use would not infringe privately owned rights. Reference heiein to any specific commercial product, process, or service by trade name, trademark, manufacturer, or otherwise does not necessarily constitute or imply its endorsemeni, recommendation, or favoring by the United States Government or any agency thereof. The vitws and opinions of authors expressed herein do not necessarily state or reflect those of the United States Government or any agency thereof.

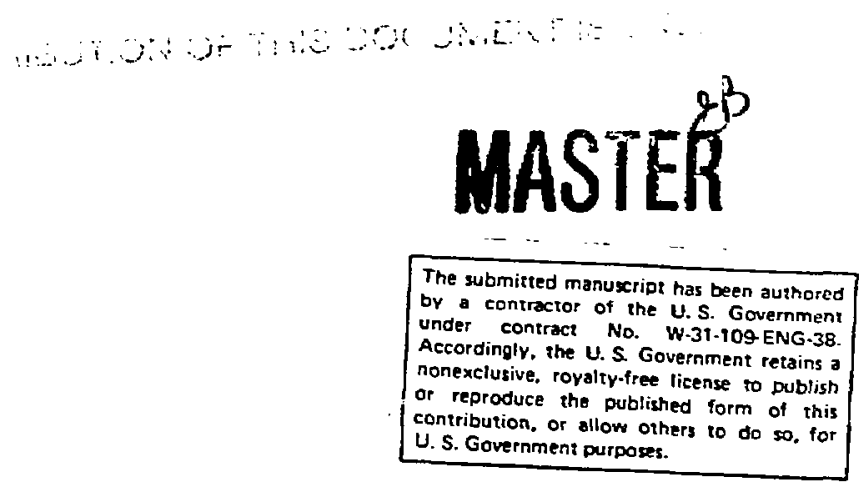




\section{Introduction}

The ATLAS accelerator is operated as a National User Facility at Argonne National Laboratory. The research program is primarily directed at investigating the dynamics of heavy-ion collisions and the structure of nuclei at high spin and temperature. The accelerator is used less frequently for other programs, including accelerator mass spectroscopy and the atomic physics of highly-charged ions. Outside users are involved in more than $2 / 3$ of all experiments.

The accelerator has existed in its' present configuration since 1985 when a major addition to the accelerator and experimental facilities was completed [1]. The linac itself consists of 7 cryostats containing 42 superconducting splitring resonators of the Argonne design [2]. Five additional resonators in individual cryostats are used as buncher/rebunchers. The injector, a tandem electrostatic accelerator, is a conventional machine except for the use of NEC straight tubes and a Pelletron charging system [3]. This configuration provides stable, reliable, low ripple operation. The accelerator tubes have been in use for 12 years, have logged over 57,000 hours of operation, and have never failed or shown any evidence of deterioration, despite being routinely operated at $108 \%$ of rated gradient. The ion source system is located on a platform allowing up to $300 \mathrm{keV}$ of acceleration[4] and can alternately accept one of several types of sputter negative-ion sources. Most frequently used are a NEC SNICSII source[5] and an Argonne modified version of the FSU inverted sputter source [6-7]. A dual-frequency, three-harmonic, gridded buncher is used at 12 $\mathrm{MHz}$ or $48 \mathrm{MHz}$ to initially bunch the $\mathrm{DC}$ beam before injection into the tandem. After the tandem, a sinusoidal parallel plate chopper is used to remove tails from the beam. Because the rigidity of heavy ions from the tandem is often greater than the bending power of the analyzing magnet $\left(\mathrm{ME} / \mathrm{q}^{2}=52 \mathrm{MeV}\right.$-amu), the second stripper is placed upstream of the magnet. The chopper then also functions as a velocity filter to remove the unwanted charge states produced by the first stripper before they reach the second stripper. This is especially important for the higher charge states where $\triangle B / B$ for different charge-state combinations $\left(q_{1}, q_{2}\right)$ is less than the resolving power of the magnet. A driven beam-sweeper is available to remove selected beam pulses for experiments with long-lived reaction products or long detector transit times, or to select an 
arbitrary duty factor [8]. A second bunching stage, this time by a split-ring superconducting resonator, is used to match the beam longitudinally into the linac.

The beam can be delivered into one of two target areas. Each experimental area is equipped with an Enge split-pole spectrograph, a large scattering chamber, and general-purpose beamlines. The Area III target room also contains a Compton-suppressed BGO/GeLi detector array for gamma spectroscopy, and a dedicated beamline for the atomic physics program. A new target area, currently under construction, will and two additional beamlines: a fragment-mass-analyzer (FMA) to separate reaction products from the primary beam is being built, and a solenoid electron-positron spectrometer is proposed. Figure 1 shows a layout of the entire facility.

\section{Resonator operating experience}

The niobium superconducting linac technology has proven to be extremely reliable, so machine interruptions for maintenance of cryogenic components are rare. The durability and ease of operation of the split-ring resonators has been particularly satisfactory. On-line performance of many resonators has remained essentially unchanged for over a decade. A cryostat will typically operate for 4 or 5 years without being taken offline. Over this time period a small number of faults will naturally accumulate, but during 12 years of operation, only a very few resonators have been unusable for extended periods. The resonators have also proven to be highly resistant to abuse. Even cryostat vacuum accidents normally require only a cycle to room temperature and a low-temperature bake at $90^{\circ} \mathrm{C}$ to revive a resonator. Multipacting conditioning is easy and fast. Barriers can invariably be conditioned away at 4.5K with only a 100-watt RF amplifier, usually in less than 30 minutes. They do not generally reoccur after the first few weeks of operation unless the cryostat is warmed above $80 \mathrm{~K}$. In storage, a dust-free environment is all that is required to maintain operating field levels; and if particulate or hydrocarbon contamination does occur, a simple solvent and deionized water rinse will restore performance. In a few cases of serious contamination, a rinse has not been effective, but the resonators were recovered by an electropolishing treatment.

Long-term use has identified two failure modes which can occur after repeated thermal cycling of the resonators. In one, the ceramic insulator which 
electrically isolates the 77K fast tuner has separated allowing the capacitive tip to float thermally. This results in an excessive heat load on the resonator. In the other, cracks have appeared at the copper-niobium interfaces of the capacitive pickup and fast-tuner ports, reducing the $Q$. Both of these problems have affected a relatively small number of resonators, but left unattended, would probably occur to the remainder over time. Repair methods and preventive modifications have been developed for both types of failure. The fast tuner has been redesigned to use an inductive coupler, eliminating the need for the ceramic isolator. The new system has been developed using PIN diodes operating in a bath of liquid nitrogen, with the diodes being controlled by a highvoltage YMOS FET driver [9]. The new system has operated at reactive power levels above $20 \mathrm{KVA}$, a factor of four increase over the earlier design. This will allow the tuning range of the resonators to be enlarged, and allow the accelerating field levels to increase. This system has been used successfully on the first cryostat of the PII and the high-voltage drivers have been installed in cryostat 6 of ATLAS. In the case of the port failure, simply etching away additional copper near the port relieves the stress on the niobium during cocling and warming. Both of these modifications will be performed on all of the existing resonators as opportunities arise over the next few years.

Last year, the maximum beam energy available was $16 \mathrm{MeV} / \mathrm{A}$ for light ions down to 3-4 MeV/A for the heaviest ions. The operating levels of each resonator in the linac for each of the experiments during the last six months of this period are plotted in Figure 2. A considerable range of operating field levels were used for each resonator, depending on the varying needs of the experiment and the condition of the resonator at the time. Although individual resonators are typically operated in a test cryostat with a 4-MV/m accelerating field at a power input of 4 watts before installation into the accelerator, on-line operation is somewhat below this level. Three resonators, R14, R27, and R41, were inoperable during the six-month period plotted, and a fourth, R42, was inoperable during part of the period. The remaining resonators were often operated at conservative field levels consistent with the demands of the experiment, so a simple average field level is not very meaningful. A more representative average can be computed by using the mean of the maximum field obtained for each resonator during the entire period. If this is done 
(excluding the three inoperable resonators), an average field of $2.83 \mathrm{MV} / \mathrm{m}$ is obtained for the entire linac.

\section{He refrigerator operating experience}

The He refrigeration system of the linac has operated very smoothly and requires very little effort for maintenance or operation. It typically runs urattended during long periods and only requires occasional tuning for changes in load. Contamination has not been a problem unless the system is exposed to air during the installation of a new branch distribution line. When contamination does occur, the capacity is reduced somewhat below the nominal 600 watts, but usually this does not present a major problem since the accelerator is frequently operating below it's maximum possible effective voltage. The contamination is easily removed by warming the contaminated section.

The flexibility of using multiple refrigerators cannot be over-emphasized. Although some efficiency and cost saving might be realized with a single large refrigerator, multiple smaller refrigerators allow operations to be maintained, albeit with a loss of cooling power, while one is warmed for cleaning or repair. Usually the experimental schedule can be organized so that no loss of beam time is experienced. Over the past year, the ATLAS accelerator has operated with three refrigerators: CTI models 2800,1630 , and 1400. Early in the summer of 1989 , a second CTI 2800 will replace the 1400 , adding $100 \mathrm{~W}$ to the total.

\section{Linac tuning}

Determining the phases of linac resonators for a new beam has, in the past, been a time consuming process. The linac is tuned empirically, by sequentially varying the phase of each of 42 resonators, while monitoring the energy of the beam with a silicon surface-barrier detector. Although, in principle, the required phases could be calculated, in practice, the results were always quite unpredictable. Recently, a concerted effort to understand the roots of this problem has made a marked improvement in the reproducibility of tunes and in the ability to calculate and set phase offsets. By continuously monitoring the phases of resonators, it was determined that phase shifts relative to the master references were occurring. The reference system had not initially been designed to accommodate as large a number of :esonant cavities or as many 
frequencies as had come into use over the past decade, but had grown organically. Some branches were capable of shifting relative to others, and signal isolation was insufficient. A new system using a bus of phase-stabilized cable and semi-rigid coax drops was installed, and immediate improvements were apparent. Previous beam setup configurations, stored in a computer file, can now be reliably recalled for use weeks or months after the initial tune-up. The tunes remain valid as long as the linac does not suffer any large disruption in the distances between resonators or in the amplitude of the accelerating fields. This improvement has significantly decreased the time required for linac setup.

\section{Beam properties}

The linac preserves the transverse and longitudinal beam quality of the injected beam. The transverse phase space is dominated by the requirement for terminal foil stripping in the tandem and an emittance between $1-3 \pi \mathrm{mm}-\mathrm{mr}$ is normally achieved, yielding beam spot sizes of $\approx 2 \mathrm{~mm}$ diameter at the target. The longitudinal emittance for heavy ions is largely determined by the contributions from the non-isochronous bend of the tandem energy analyzer, and for doubly stripped beams, the energy spread introduced by the second stripper. Measurements of the longitudinal phase space of the beam at the target have shown the beam emittance to be $10-30 \pi \mathrm{keV}$-ns for singly-stripped ions, and $30-50 \pi k \in V-n s$ for doubly-stripped ions. This allows rebunching the beam at the target to very narrow pulses, $100-200$ ps, or alternatively, debunching to $20-100 \mathrm{keV}$. A resonant TOF energy measurement system provides relative energy determinations with a precision of $\Delta E / E \approx 10^{-4}$ and absolute measurements with an accuracy of $10^{-3}$. Long-term stability of the beam energy is measured to be better than $\Delta E / E=3 \times 10^{-4}$.

The ATLAS accelerator has been used to accelerate a wide variety of ion species ranging from ${ }^{6} \mathrm{Li}$ to ${ }^{127} \mathrm{I}$. Figure 3 is a histogram of the beam usage during 1988. The selection of ions is a function of the research program, the availability of cumplementary nuclear targets, and the capability of the accelerator, ion source, and injection system. As much as $10 \%$ of the research used beams with $A \geq 79$, and slightly more than $1 / 3$ of the beam time was with ions with $A \geq 58$. The sulfur isotopes, $A=32,34$, and 36 , constituted another $1 / 3$, 
and beams with $A \leq 29$ were used the remaining $1 / 3$ of the time. A trend to the use of the heavier isotopes has been clearly evident over recent years.

\section{Conclusions}

A decade of experience has proven the technology of niobium superconducting heavy ion linacs to be reliable, easy to operate, and easy to maintain. Each year since the completion of ATLAS, the number of hours available for research has been able to be increased. Table $y$ itemizes the consumption of accelerator time by category for fiscal year 1988 . Operations statistics show the cryogenic components of the accelerator system to be no more prone to failure than the conventional tandem electrostatic accelerator used as an injector. Although achieving accelerating fields oniine equal to the fields seen in off-line tests continues to be a challenge, methods of gaining improvements are known and can be implemented with existing technology. At Argonne, confidence in the superconducting linac technology of ATLAS is now leading to the development of a superconducting injector accelerator composed of an electron cyclotron resonance ion source injecting into a new linac section, optimized for ions with velocities as low as $\beta=0.009$ [10-11]. In the latter part of 1989, this Positive Ion Injector (PII) will begin to supplant the tandem electrostatic machine as an injector into ATLAS. 
Figure Captions

Figure 1. Layout of the ATLAS accelerator facility.

Figure 2. The operating levels of the ATLAS resonators over a six-month period. Each point represents the operating level of a resonator for a particular experiment. $E_{a}$ is the average accelerating field for a synchronous particle averaged over the total length of the resonator. The resonator ID indicates the position in the linac, e.g. R34 is the fourth resonator in cryostat 3. The shaded areas indicate the three classes of resonators used in the linac which are optimized for synchronous particles with velocities $\beta=0.06,0.10$, and 0.16 , respectively. The solid line is the average operating level as defined in the text.

Figure 3. Distribution of beam time during 1988 by isotope. 
Table 1

Distribution of ATLAS accelerator time during FY 1988.

Research

Machine studies, setup and tuning

Scheduled maintenance

Unscheduled maintenance
Hours Percent of year $3648 \quad 42 \%$ $1486 \quad 17 \%$

$550 \quad 5 \%$

$912 \quad 10 \%$ 
[1] J. Aron, et al., Rev. Sci Instrum. 57, 737 (1986).

[2] K. W. Shepard, IEEE Trans. Nucl. Sci. NS-26, p. 3659 (1979).

[3] J. L. Yntema, P. K. Den Hartog, W. Henning and W. Kutschera, Nucl. Instrum. and Meth. 184,223 (1981).

[4] E. Minehara, W. Kutschera, P. Den Hartog, P. Billquist, and Z. Liu, Rev. Sci. Instrum. 57 , 742 (1986).

[5] G. T. Caskey, R. A. Douglas, H. T. Richards and H. V. Smith, Jr., Nucl. Instrum. and Meth. 157. 1 (1978).

[6] K. R. Chapman, IEEE Trans. Nucl. Sci. NS-23, p. 1109 (1976).

[7] P. J. Billquist and J. L. Yntema, Nucl. Instrum. and Meth. 178, 9 (1980).

[8] J. M. Bogaty, Proceedings of the 1989 IEEE Particle Accelerator Conf., Chicago, II., 20-23 March 1989 (to be published).

[9] J. M. Bogaty, B. E. Clifft, K. W. Shepard and G. P. Zinkann, Proceedings of the 1989 IEEE Particle Accelerator Conf., Chicago, II., 20-23 March 1989 (to be published).

[10] R. C. Pardo, L.M. Bollinger and K. W. Shepard, Nucl. Instrum. and Meth. B24/24, 746 (1987).

[11] P.K. Den Hartog, et al., Nucl. Instrum. and Meth. B40/41, 900 (1989). 


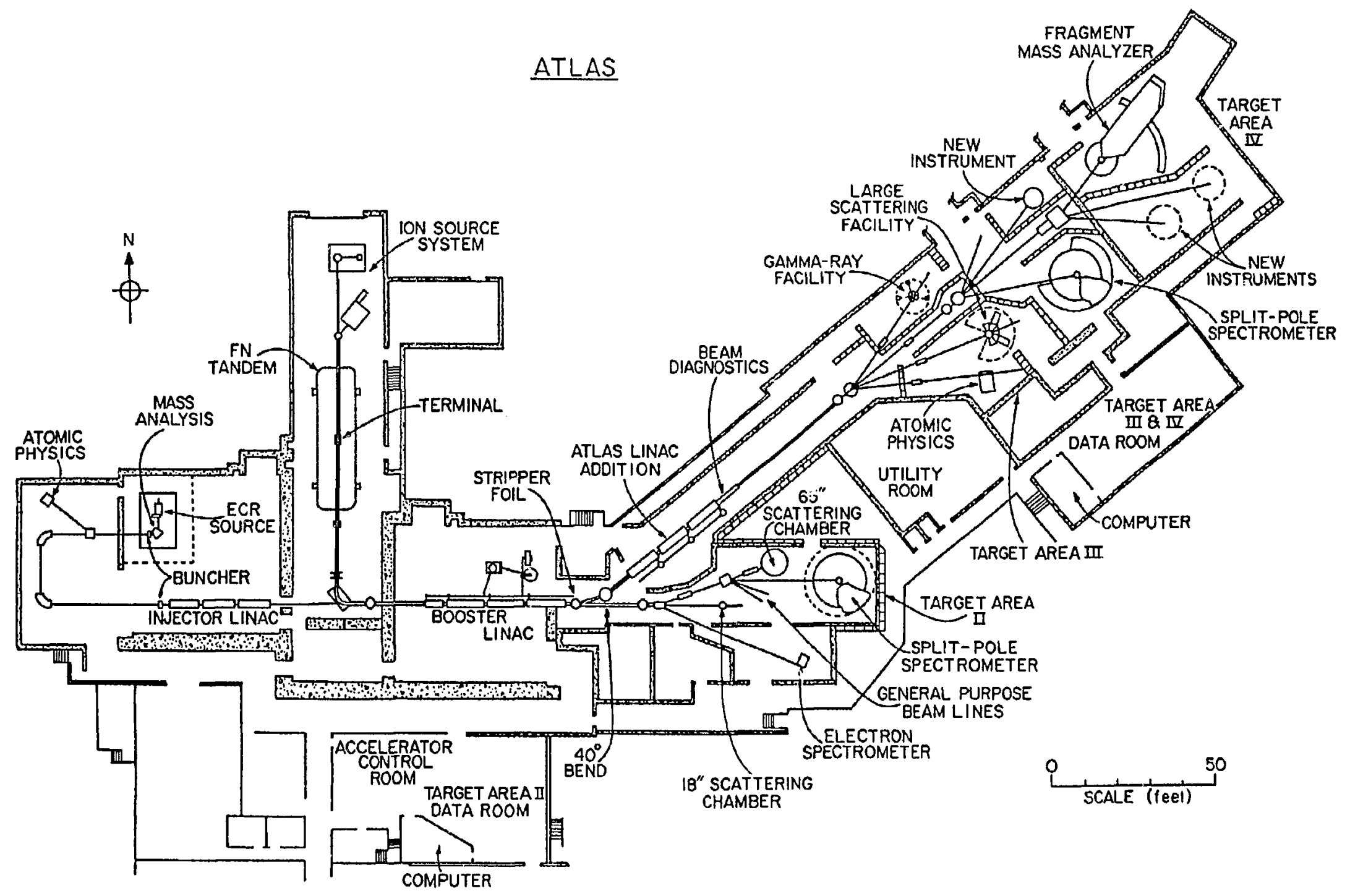




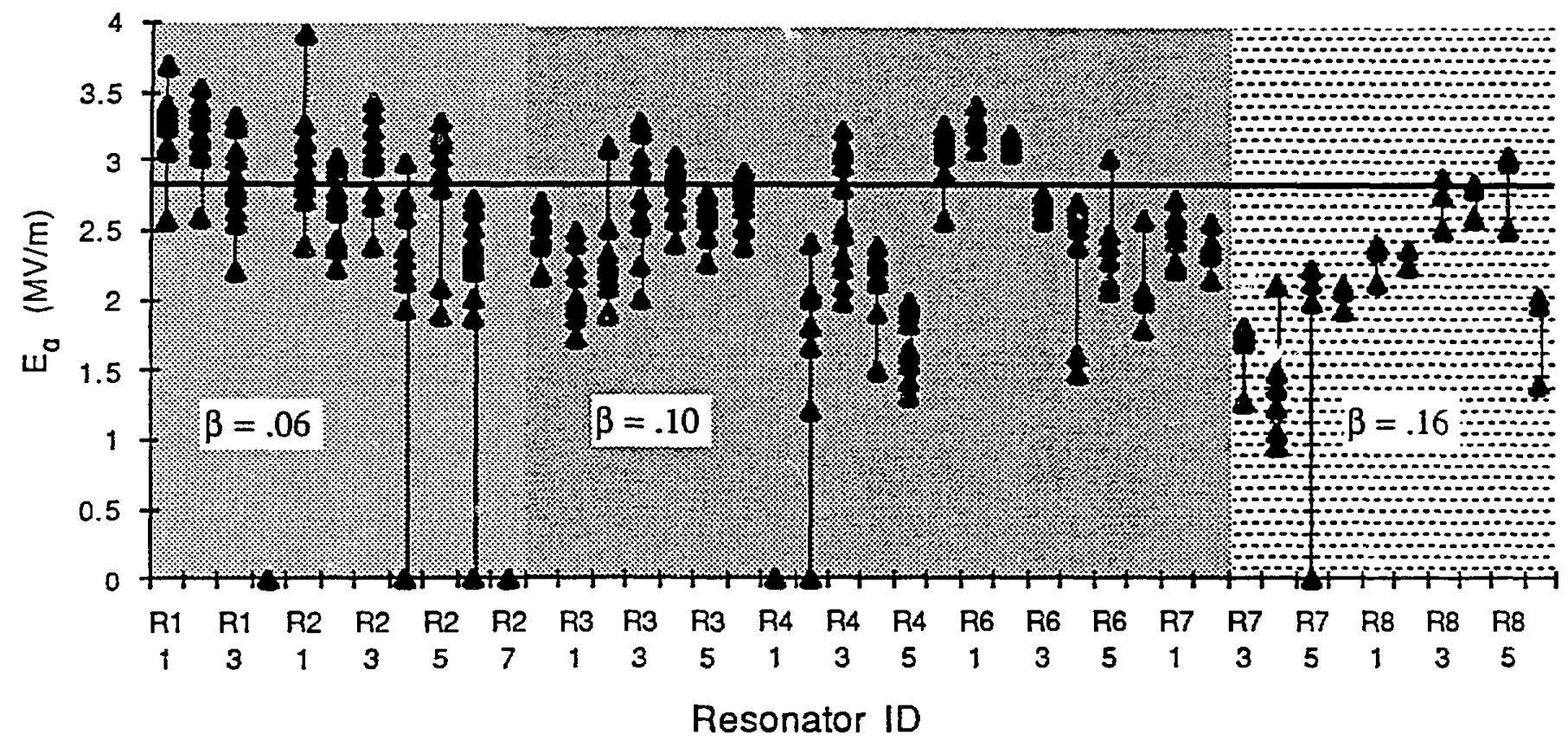




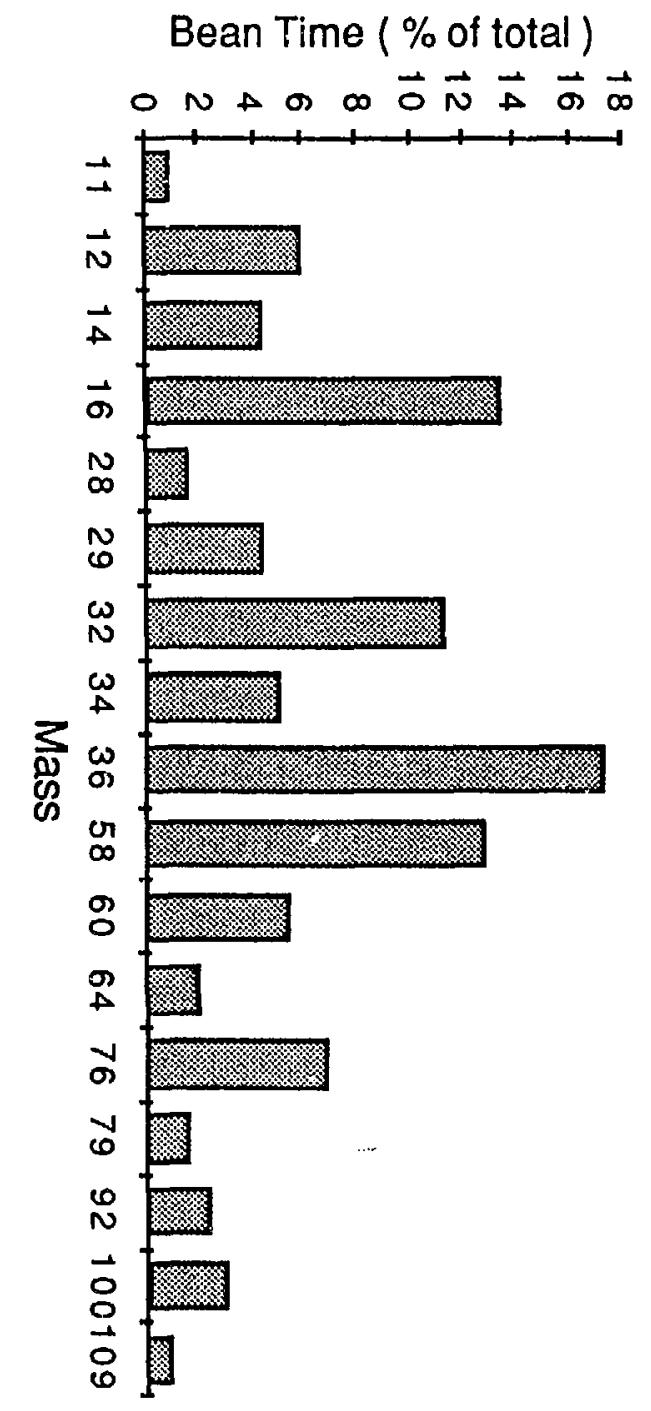

